\title{
Rising Mortality Rate of Cervical Cancer in Younger Women in Urban China
}

\author{
Min Wei, MD ${ }^{1,2}$, Wei Zhou, $M S^{2}$, Yongyi Bi, PhD², Hong Wang, PhD'2, Yu Liu, PhD ${ }^{3}$, and Zhi-Jiang Zhang, PhD ${ }^{2}$ \\ 'Department of Obstetrics and Gynecology, Renmin Hospital of Wuhan University, Wuhan, China; ${ }^{2}$ Department of Preventive Medicine, School of \\ Health Sciences, Wuhan University, Wuhan, China; ${ }^{3}$ Department of Statistics and Management, School of Management, Wuhan Institute of \\ Technology, Wuhan, China.
}

BACKGROUND: In recent decades, much effort has been made in China to reduce the burden of cervical cancer. OBJECTIVE: Our study's purpose was to examine trends of cervical cancer mortality in each 5-year age group for urban and rural Chinese women, respectively.

DESIGNS: Retrospective analysis of cervical cancer mortality from 1987 to 2015 from the World Health Organization Cancer Mortality Database and China Health Statistical Yearbooks.

PARTICIPANTS: Chinese women.

MAIN MEASURES: Trends were examined using annual percent change (APC) and average annual percent change (AAPC) via Joinpoint regression models for each 5-year age group in urban and rural areas, respectively.

RESULTS: In urban China, mortality rate of cervical cancer increased significantly among urban women aged 2554 years (AAPC 2.12 5.49\%), in contrast to a decline trend among urban women older than 60 years (AAPC $3.61 \sim-5.35 \%$ ). In rural China, cervical cancer rates declined in all age groups, but the magnitude was smaller in women aged 30-54 years (AAPC - 0.59 - 2.20\%) compared to women older than 55 years (AAPC - 3.06 $4.33 \%)$.

CONCLUSION: Mortality rate of cervical cancer is rising at an alarming rate in younger women in urban China. Timely intervention is required for these vulnerable populations.

KEY WORDS: cervical cancer; disparity; age-specific mortality; trend.

J Gen Intern Med 34(2):281-4

DOI: $10.1007 / \mathrm{s} 11606-018-4732-\mathrm{Z}$

(c) Society of General Internal Medicine 2018

\section{INTRODUCTION}

Cervical cancer is the fourth most common cancer and the fourth leading cause of cancer-related death among women worldwide. ${ }^{1,2}$ Approximately 527,600 women are newly diagnosed with cervical cancer each year with 265,700 cervical cancer deaths annually worldwide. ${ }^{1}$ Its mortality rate is

Electronic supplementary material The online version of this article (https://doi.org/10.1007/s11606-018-4732-z) contains supplementary material, which is available to authorized users.

Received June 16, 2018

Revised September 11, 2018

Accepted October 25, 2018

Published online November 27, 2018 especially high in developing countries. ${ }^{1,2}$ In China alone, it is estimated that 98,900 cervical cancer cases, accounting for approximately $20 \%$ of total new cases globally, and nearly 30,500 cervical cancer deaths occur each year. ${ }^{3}$

Cervical cancer is one of the most preventable forms of cancer. There has also been significant improvement in screening, ${ }^{4-8}$ diagnosis, ${ }^{9-11}$ treatment, ${ }^{12-15}$ and even chemoprevention $^{16-18}$ for cervical cancer. In the past decade, much effort has been made in China to reduce the burden of cervical cancer. Since 2009, free cervical cancer screening has been available in some rural regions in China under governmentsponsored programs. ${ }^{19}$ On the other hand, however, the prevalence of human papillomavirus (HPV) infection remains at a high level in both urban and rural China. ${ }^{20,21}$

Our study's purpose was to examine trends in cervical cancer mortality from 1987 to 2015 in China, among each 5year age group, comparing urban and rural rates.

\section{METHODS}

\section{Data Source}

We extracted cervical cancer mortality data in China from 1987 to 2000 from the World Health Organization Cancer Mortality Database (International Agency for Research on Cancer, Lyon, France). Data were coded using International Classification of Disease (ICD-9) classification and tabulated in 5-year age groups $(20 \sim, 25 \sim \ldots 80 \sim, 85 \sim)$. In addition, the location (urban or rural) was collected. From 2003 to 2015, cervical cancer mortality data for the same groups were extracted from China Health Statistical Yearbooks using ICD-10 codes. These two databases are operated by the Center for Health Information and Statistics (CHIS) and have been recognized as nationally representative for common cancers. ${ }^{22-24}$ Because mortality in 2001 was not available and 2002 mortality rates were not deemed credible due to the transition from ICD-9 to ICD-10, we imputed the data for these two years by interpolation.

\section{Statistical Analysis}

Poisson regression was used to analyze the association between area (urban vs. rural area), calendar year, age (5-year increment in age), and the risk of cervical cancer mortality. As 
the Lagrange multiplier test showed the data were overdispersed $(p<0.01)$, the analysis was corrected by negative binomial regression (SAS Institute, V 9.1, Cary, NC).

Joinpoint regression analysis was used to quantitatively describe the trends of cervical cancer mortality for each 5year age group $(20 \sim, 25 \sim, 30 \sim \ldots 80 \sim, 85 \sim)$ in urban and rural areas, respectively. A maximum of four joinpoints was allowed. The average annual percent changes (AAPC) and associated $95 \%$ confidence interval (CI) were calculated to measure the direction and magnitude of trends over the full range of periods between 1987 and 2015 periods. Annual percent changes (APC) and associated 95\% CI were calculated to measure the direction and magnitude of trends during each corresponding time interval. These analyses were performed using the Joinpoint Regression Program (Statistical Methodology and Applications Branch, Surveillance Research Program, National Cancer Institute, V.4.5.0.1).

All statistical tests were two-sided with a $p$ value $<0.05$ considered significant.

\section{RESULTS}

\section{Linear Trend Assessment in All Age Groups}

Cervical cancer mortality had a linear decline trend, with an average annual decrease of $-3 \%(\mathrm{OR}=0.97$ each calendar year, 95\% CI 0.97 0.98, $p<0.01$; Table 1 ). The mortality rate was higher in urban than in rural areas $(\mathrm{OR}=1.30,95 \% \mathrm{CI}$ $1.20-1.40, p<0.01)$, and mortality rate increased with age $(\mathrm{OR}=1.31$ for each 5 -year increase in age, 95\% CI $1.29 \sim 1.32, p<0.01)$.

\section{Trend Analysis Stratified by 5-Year Age Groups}

In urban areas, cervical cancer mortality increased for those aged 25-54 years between 1987 and 2015, with AAPCs of 2.12 5.49\%. Of note, women aged 35-39 years experienced the highest mortality rates with an AAPC of $5.49 \%$. In contrast, among urban women, those older than 60 years experienced decreasing mortality rates, with AAPCs of -3.61 to $5.35 \%$ (Fig. 1, Supplement Table).

In rural areas, the mortality rate decreased in all age groups during the period 1987-2015. The magnitude of decline was smaller in younger than in older women: the AAPCs were 0.59 to $-2.20 \%$ among those aged $30-50$ years old, compared to -3.06 to $-4.33 \%$ among those aged $>55$ years (Fig. 1, Supplement Table).

Table 1 The Negative Binomial Regression Analysis of Risk Factors for Cervical Cancer Mortality

\begin{tabular}{lllll}
\hline & References & OR & 95\% CI & $\boldsymbol{p}$ value \\
\hline Year & Year 1987 & 0.97 & $0.97 \sim 0.98$ & $<0.01$ \\
Area & Rural area & 1.30 & $1.20 \sim 1.40$ & $<0.01$ \\
Age & Age 20 25 & 1.31 & $1.29 \sim 1.32$ & $<0.01$ \\
\hline
\end{tabular}

\section{DISCUSSION}

Our study analyzed cervical cancer mortality trends, stratified by age groups and location (urban vs. rural) from 1987 to 2015. While the overall trend demonstrated a decline in cervical cancer mortality, urban women aged 25-54 have experienced an increase in mortality, up to $5.5 \%$ annually, and rural women aged 30-50 have experience a much smaller decline magnitude than older rural women aged $>55$ years old.

Compared to the decreasing trends in rural area and in urban older women, the rising trend of cervical cancer mortality in urban younger women is particularly noteworthy. Although we do not have data to explore the underlying reasons for this finding, there are a number of possibilities. In past few decades, China underwent rapid urbanization and women experienced a number of lifestyle changes, including younger age at first sexual intercourse, increased number of sexual partners, and an increase in smoking, ${ }^{25}$ all of which are associated with higher risk of HPV infection. It is likely that these lifestyle changes are more common in younger urban women than in older urban women or rural women. Given that HPV infection is the most important risk factor for cervical cancer, it is likely that urban younger women experienced higher rates of HPV infection at an earlier age than urban older women and rural women. Indeed, numerous studies have reported a peak of HPV infection in urban Chinese women less than 25 years in age. ${ }^{21,26-31} \mathrm{HPV}$ infection likely explains the rising trend of cervical cancer mortality in urban younger women.

There are two important policy implications from our study. First, there is a need to initiate national programs for vaccinating young Chinese women and men against HPV infection. Administration of HPV vaccine induces durable anti-HPV responses and prevents HPV infection. ${ }^{20,}{ }^{32}$ Currently, HPV vaccines of 2- and 4-valent are available in China, and a 9valent vaccine has been available in parts of China since May 2018. However, awareness and willingness to receive HPV vaccination remain low. ${ }^{33-35}$ Efforts are urgently required to improve Chinese vaccination rates. Secondly, there is the need to improve better utilization of cervical cancer screening in both urban and rural areas. Availability of screening is in a large part affected by the national health care system. Rural women aged 35 to 59 years benefit from a free cervical cancer screening program (the National Cervical Cancer Screening Program in Rural Areas, NCCSPRA) since 2009. ${ }^{19}$ It is estimated that 11.7 million rural women between 35 and 59 years old from 221 pilot counties accessed this screening program within 3 years after its inception and approximately 10 million annually thereafter. In contrast, cervical cancer screening services have not been widely utilized in urban area in China. In 2010, only $29.1 \%$ of urban women reported a history of a Papanicolaou test. ${ }^{36}$ There may be two possible reasons for the underutilization of this service in urban China. One may be the low awareness of cervical cancer screening among urban women. Another reason may be that screening services in urban area are provided by hospitals and Maternal and Child Care Centers, 

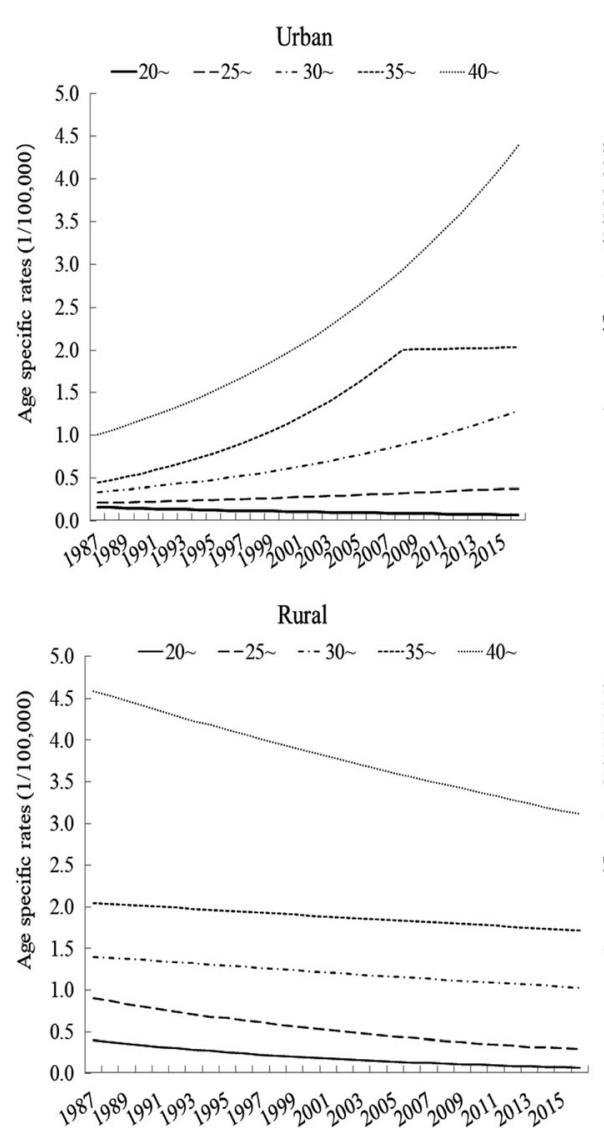
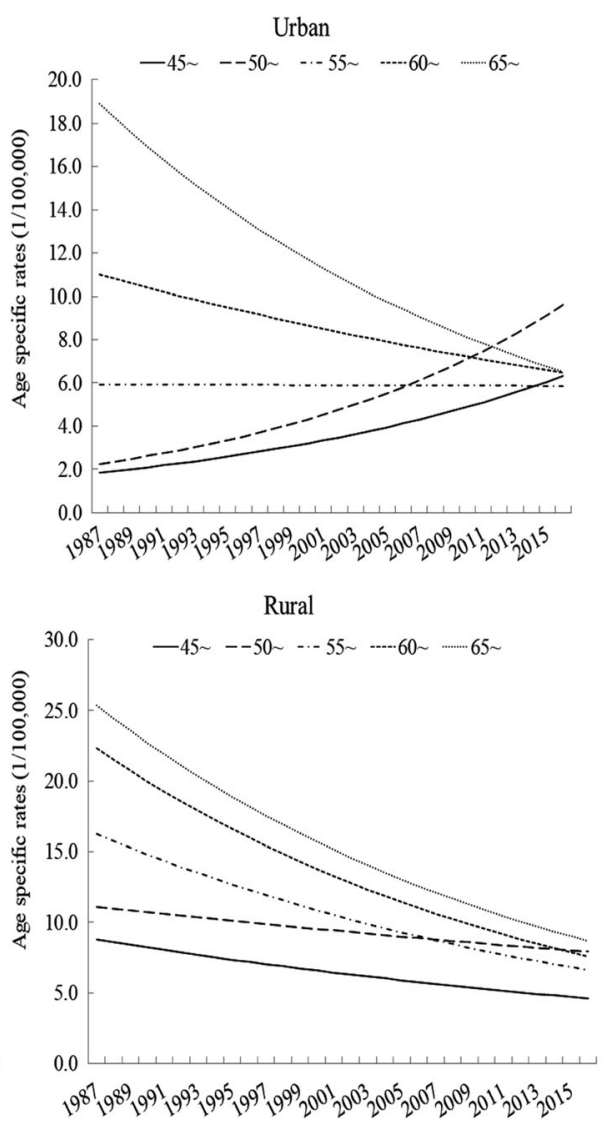

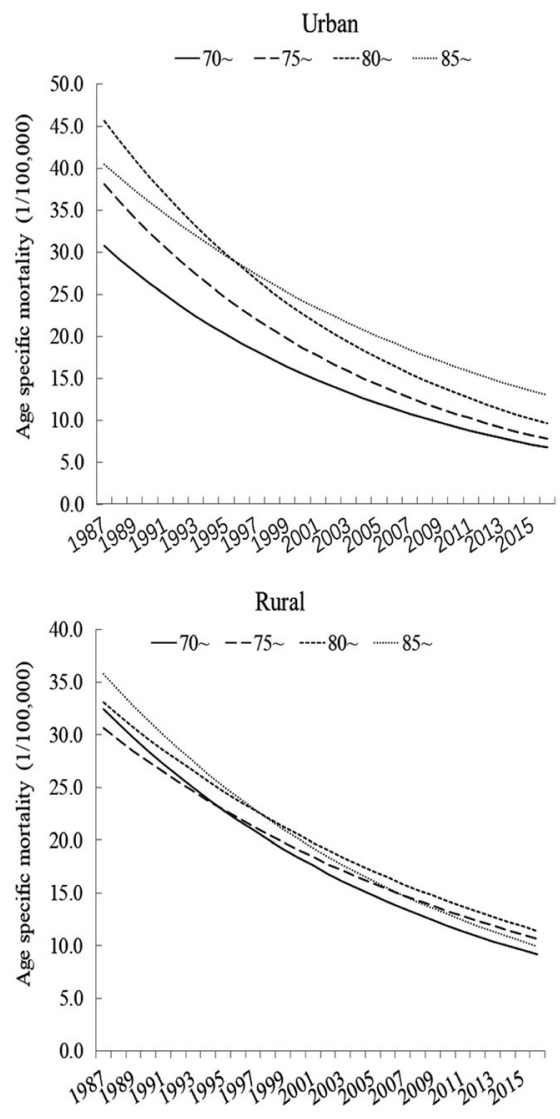

Figure 1 Annual changes in cervical cancer mortality rates for Chinese women from 1987 to 2015, stratified on each 5-year age group and urban/rural area.

which are free to very limited population dependent on the local policy or funding. Greater availability and advertisement of the importance of screening are needed.

A study strength was our examination of mortality rates for each 5-year age group, which enables us to find differential trends between younger and older women. However, our study has several limitations. First, there was large fluctuation in rates in rural areas, because of population mobility and inferior data quality from these areas. In contrast, mortality rates in urban areas were relatively stable. Second, as urbanization progresses in China, classification of urban and rural areas may have changed during the study period for some sampling sites.

In conclusion, we found that cervical cancer is rising at an alarming rate among younger urban women. In rural China, mortality rate is declining with a lower rate in younger than in older women. Measures are urgently needed to control the burden of cervical cancer in these populations.

Corresponding Author: Zhi-Jiang Zhang, $\mathrm{PhD}$; Department of Preventive Medicine, School of Health Sciences Wuhan University, Wuhan 430071, China (e-mail: zhang22968@163.com).

Authors' Contributions Min Wei drafted the manuscript; Wei Zhou drafted the manuscript and collected and analyzed data; Yongyi Bi, Hong Wang, and Yu Liu edited and commented the manuscript; and Zhi-Jiang Zhang concepted the study, supervise the research, and edited the manuscript.
Funding The work was financially supported by the National Natural Science Foundation of China (grant number 81641123) and the Fundamental Research Funds for the Central Universities (grant number 2042017kf0193).

\section{Compliance with Ethical Standards:}

Conflict of Interest: The authors declare that they do not have a conflict of interest.

\section{REFERENCES}

1. Torre LA, Islami F, Siegel RL, Ward EM, Jemal A. Global Cancer in Women: Burden and Trends. Cancer Epidemiol Biomark Prev 2017; 26: 444-457.

2. Fidler MM, Gupta S, Soerjomataram I, Ferlay J, Steliarova-Foucher E, Bray F. Cancer incidence and mortality among young adults aged 2039 years worldwide in 2012: a population-based study. Lancet Oncol 2017; 18(12): 1579-1589.

3. Chen W, Zheng R, Baade PD, et al. Cancer statistics in China, 2015. CA Cancer J Clin 2016; 66: 115-132.

4. Giao YL, Sellors JW, Eder PS, et al. A new HPV-DNA test for cervicalcancer screening in developing regions: a cross-sectional study of clinical accuracy in rural China. Lancet Oncol 2008; 9: 929-936.

5. Zhao FH, Lin MJ, Chen F, et al. Performance of high-risk human papillomavirus DNA testing as a primary screen for cervical cancer: a pooled analysis of individual patient data from 17 population-based studies from China. Lancet Oncol 2010; 11: 1160-1171. 
6. Pan QJ, Hu SY, Zhang X, et al. Pooled analysis of the performance of liquid-based cytology in population-based cervical cancer screening studies in China. Cancer Cytopathol 2013; 121: 473-482.

7. Dong $\mathbf{L}$, Wang $\mathbf{M Z}, \mathbf{Z h a o} \mathbf{X L}$, et al. Human papillomavirus viral load as a useful triage tool for non-16/18 high-risk human papillomavirus positive women: A prospective screening cohort study. Gynecol Oncol 2018; 148(1): 103-110.

8. Zheng $\mathbf{B}$, Yang $\mathbf{H}, \mathbf{L i} \mathbf{Z}$, et al. HPV test results and histological follow-up results of patients with LSIL Cervical Cytology from the Largest CAPcertified laboratory in China. J Cancer 2017; 8: 2436-2441.

9. Lv XL, Hou M, Duan XJ. Correlation analysis between the parameters of contrast-enhanced ultrasonography in evaluating cervical cancer metastasis and expression of E-cadherin. Oncol Lett 2017; 14: 4641-4646.

10. Wei L, Gan $\mathbf{Q}$, Ji T. Cervical cancer histology image identification method based on texture and lesion area features. Comput Assist Surg (Abingdon) 2017; 22: 186-199.

11. Li X, Wang $\mathbf{L}, \mathbf{L i} \mathbf{Y}$, Song $\mathbf{P}$. The Value of Diffusion-Weighted Imaging in Combination With Conventional Magnetic Resonance Imaging for Improving Tumor Detection for Early Cervical Carcinoma Treated With Fertility-Sparing Surgery. Int J Gynecol Cancer 2017; 27: 1761-1768.

12. Zhang ZJ, Li S. The prognostic value of metformin for cancer patients with concurrent diabetes: a systematic review and meta-analysis. Diabetes Obes Metab 2014; 16: 707-710.

13. Wu SG, Zhang WW, Sun JY, Li FY, He ZY, Zhou J. Multimodal treatment including hysterectomy improves survival in patients with locally advanced cervical cancer: A population-based, propensity score-matched analysis. Int J Surg 2017; 48: 122-127.

14. Qi YX, Liu K, Yin J, Li L. Evaluation of short- and long-term efficacy of chemoradiotherapy for advanced cervical cancer using HSP70 protein combined with multimodal MRI. J Cell Biochem 2018; 119(4): $3017-$ 3029 .

15. Lei H, Gui D, He Y. Short- and long-term outcomes of laparoscopic radical hysterectomy for obese patients with cervical cancer. J BUON 2017; 22: 958-965.

16. Zhang ZJ, Bi Y, Li S, et al. Reduced risk of lung cancer with metformin therapy in diabetic patients: a systematic review and meta-analysis. Am J Epidemiol 2014; 180: 11-14.

17. Zhang ZJ, Zheng ZJ, Kan H, et al. Reduced risk of colorectal cancer with metformin therapy in patients with type 2 diabetes: a meta-analysis. Diabetes Care 2011; 34: 2323-2328.

18. Zhang ZJ, Zheng ZJ, Shi R, Su Q, Jiang Q, Kip KE. Metformin for liver cancer prevention in patients with type 2 diabetes: a systematic review and meta-analysis. J Clin Endocrinol Metab 2012; 97: 2347-2353.

19. Women's health in rural China. Lancet 2009; 374: 358.

20. Li WJ, Xu HX, Chen ZH, Xu WD, Wu YJ. Characteristics of carcinogenic human papillomavirus infection in Suzhou: Epidemiology, vaccine evaluation, and associated diseases. J Med Virol 2017; 89: 895-901.
21. Xu HH, Lin A, Chen YH, et al. Prevalence characteristics of cervical human papillomavirus (HPV) genotypes in the Taizhou area, China: a cross-sectional study of 37967 women from the general population. BMJ Open 2017; 7: e014135.

22. Liu X, Bi Y, Wang $\mathbf{H}$, et al. Different Trends of Mortality from Colorectal Cancer between Age Groups in China: An Age-Period-Cohort and Joinpoint Analysis. Public Health 2018; 166: 45-52.

23. Wang $\mathbf{L}$, Yu C, Liu Y, et al. Lung Cancer Mortality Trends in China from 1988 to 2013: New Challenges and Opportunities for the Government. Int J Environ Res Public Health 2016; 13: 1025.

24. Wang $\mathbf{P}, \mathbf{X u} \mathbf{C}, \mathbf{Y u} \mathbf{C}$. Age-period-cohort analysis on the cancer mortality in rural China: 1990-2010. Int J Equity Health 2014; 13: 1.

25. Miao $\mathbf{J}, \mathbf{W u} \mathbf{X}$. Urbanization, socioeconomic status and health disparity in China. Health Place 2016; 42: 87-95.

26. Li Z, Liu F, Cheng S, et al. Prevalence of HPV infection among 28,457 Chinese women in Yunnan Province, southwest China. Sci Rep 2016; 6: 21039.

27. Shen Y, Gong JM, Li Y Y, et al. Epidemiology and genotype distribution of human papillomavirus (HPV) in women of Henan Province, China. Clin Chim Acta 2013; 415: 297-301.

28. Wang $\mathbf{R}$, Guo XL, Wisman GB, et al. Nationwide prevalence of human papillomavirus infection and viral genotype distribution in 37 cities in China. BMC Infect Dis 2015; 15: 257.

29. Wu C, Zhu X, Kang Y, et al. Epidemiology of Humanpapilloma virus infection among women in Fujian, China. BMC Public Health 2017; 18: 95.

30. Jing $\mathbf{L}$, Zhong $\mathbf{X}$, Zhong $\mathbf{Z}$, et al. Prevalence of human papillomavirus infection in Guangdong Province, China: a population-based survey of 78,355 women. Sex Transm Dis 2014; 41: 732-738.

31. Chen Z, Wang $\mathbf{Q}$, Ding X, Li $\mathbf{Q}$, Zhong R, Ren H. Characteristics of HPV prevalence in Sichuan Province, China. Int J Gynaecol Obstet 2015; 131 : 277-280.

32. Huang T, Liu Y, Li Y, et al. Evaluation on the persistence of anti-HPV immune responses to the quadrivalent HPV vaccine in Chinese females and males: Up to 3.5years of follow-up. Vaccine 2018; 36: 1368-1374.

33. Zou H, Meng X, Jia T, et al. Awareness and acceptance of human papillomavirus (HPV) vaccination among males attending a major sexual health clinic in Wuxi, China: A cross-sectional study. Hum Vaccin Immunother 2016; 12: 1551-1559.

34. Zou H, Wang W, Ma $\mathbf{Y}$, et al. How university students view human papillomavirus (HPV) vaccination: A cross-sectional study in Jinan, China. Hum Vaccin Immunother 2016; 12: 39-46.

35. Wang $\mathbf{Z}$, Wang $\mathbf{J}$, Fang $\mathbf{Y}$, et al. Parental acceptability of HPV vaccination for boys and girls aged 9-13years in China - A population-based study. Vaccine 2018; 36: 2657-2665.

36. Wang B, He M, Chao A, et al. Cervical Cancer Screening Among Adult Women in China, 2010. Oncologist 2015; 20: 627-634. 